\title{
Recurrent Hemiparesis in a Young Woman With Hyperthyroidism
}

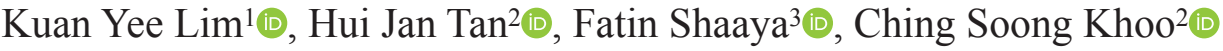

\author{
${ }^{1}$ Department of Medicine, Faculty of Medicine, Universiti Kebangsaan Malaysia Medical Centre, Kuala Lumpur, Malaysia \\ ${ }^{2}$ Neurology Unit, Department of Medicine, Faculty of Medicine, Universiti Kebangsaan Malaysia Medical Centre, Kuala Lumpur, Malaysia \\ ${ }^{3}$ Department of Radiology, Faculty of Medicine, Universiti Kebangsaan Malaysia Medical Centre, Kuala Lumpur, Malaysia
}

\begin{abstract}
A 25-year-old Chinese woman presented with a sudden onset of left-sided weakness. Previously, she had been diagnosed with Grave's disease, for which she did not follow-up. Her clinical examination revealed a diffusely swollen neck and left hemiparesis with hyperreflexia in the left upper and lower extremities, and her electrocardiogram showed sinus tachycardia. Hyperthyroidism with elevated anti-thyroxin peroxidase antibodies from her blood investigations was observed. Other blood tests, including infective screening, connective tissue disease, and thrombophilia were unremarkable, and her echocardiogram was normal. Her brain computed tomography (CT) revealed a recent infarct at the right frontal lobe (Figure $1 \mathrm{a}, \mathrm{b}$.), which was further confirmed with a brain magnetic resonance imaging (MRI) (Figure $1 \mathrm{c}$,d.). She was treated for young stroke with hyperthyroidism, and was discharged with aspirin, statin, and carbimazole.
\end{abstract}

She later presented with another two episodes of recurrent transient ischemic attacks, resulting in hemiparesis on the left side. Her clinical examination revealed a similar left spastic hemiparesis as observed in her previous presentation. Otherwise, her other systemic examinations were unremarkable. Her brain $\mathrm{CT}$ angiogram revealed a long segment stenosis involving both internal carotid arteries, severe $A_{1}$, and $M_{1}$ stenosis with collaterals around these areas. The cerebral angiography further confirmed the above findings (Figure 1). Based on her previous thyroid function test and the overall radiological findings, a diagnosis of Moyamoya disease associated with Grave's disease was established.

After a multidisciplinary discussion, she was scheduled for a revascularization procedure. After optimizing her thyroid status, she underwent a successful revascularization procedure. Neurologically, she remained stable during the surgery.
Moyamoya disease is a vasculopathy of the intracranial arteries found predominantly in the East Asian populations, particularly Chinese, Korean, and Japanese people. ${ }^{1}$ The association of Moyamoya disease with hyperthyroidism has been reported since $2009,{ }^{2}$ with no clear pathophysiology to explain the coexistence of both the diseases. Thyrotoxicosis is a trigger of cerebral ischemia in Moyamoya disease. ${ }^{2}$ Surgical revascularization after optimization of the thyrotoxicosis is crucial to prevent further ischemic events. ${ }^{3}$

Axial image of noncontrast CT Brain (Figure 1 and 2) taken at the supraganglionic level showing ill-defined hypodensity involving the $\mathrm{M}_{5}$ and $\mathrm{M}_{6}$ region of the right middle cerebral artery territory associated with sulcal effacement. MRI brain in FLAIR sequence (Figure 1c) and $\mathrm{T}_{1} \mathrm{~W}$ post gadolinium administration (Figure 1d) showed leptomeningeal enhancement and hyperintense signal of the right paracentral, supramarginal, and angular gyri (arrowheads) giving rise to ivy sign appearance likely due to the engorged pia vessels with thickened arachnoid membrane. Right internal carotid artery (ICA) angiography (Figure $2 \mathrm{a}, \mathrm{b}$.) showed multiple lenticulostriate collaterals giving the "puff of smoke" appearance (arrowheads), severe stenosis of the right supraclinoid ICA and ICA bifurcation (thin arrows), and small caliber right $\mathrm{M}_{1}$ (thick arrow). Left ICA angiography (Figure $2 \mathrm{c}$,d.) showed left ICA small in caliber throughout, multiple lenticulostriate collaterals giving the "puff of smoke" appearance (asterisk), stenosis of left supraclinoid ICA and ICA bifurcation (thin arrows), and stenotic left $\mathrm{M}_{1}$ (thick arrow) and left ACA (arrowhead). Left vertebral angiogram (Figure 2e) showed stenotic $\mathrm{V}_{4}$ and basilar artery (arrowhead) with multiple collaterals of the vertebrobasilar system (asterisks).

Corresponding author: Ching Soong Khoo, Neurology Unit, Department of Medicine, Faculty of Medicine, Universiti Kebangsaan Malaysia Medical Centre, Kuala Lumpur, Malaysia

e-mail: chingsoongkhoo@gmail.com

Received: September 3, 2021 Accepted: November 9, 2021 Available Online Date: January 18, 2022 • DOI: 10.4274/balkanmedj.galenos.2021.2021-8-144

Available at www.balkanmedicaljournal.org

ORCID iDs of the authors: K.Y.L. 0000-0003-2671-3722; H.J.T. 0000-0003-4827-7984; F.S. 0000-0002-6433-1138; C.S.K. 0000-0002-6756-0411.

Cite this article as:

Lim KY, Tan HJ, Shaaya F, Khoo CS. Recurrent Hemiparesis in a Young Woman With Hyperthyroidism. Balkan Med J.; 2022; 39(1):68-70.

Copyright@Author(s) - Available online at http://balkanmedicaljournal.org/ 


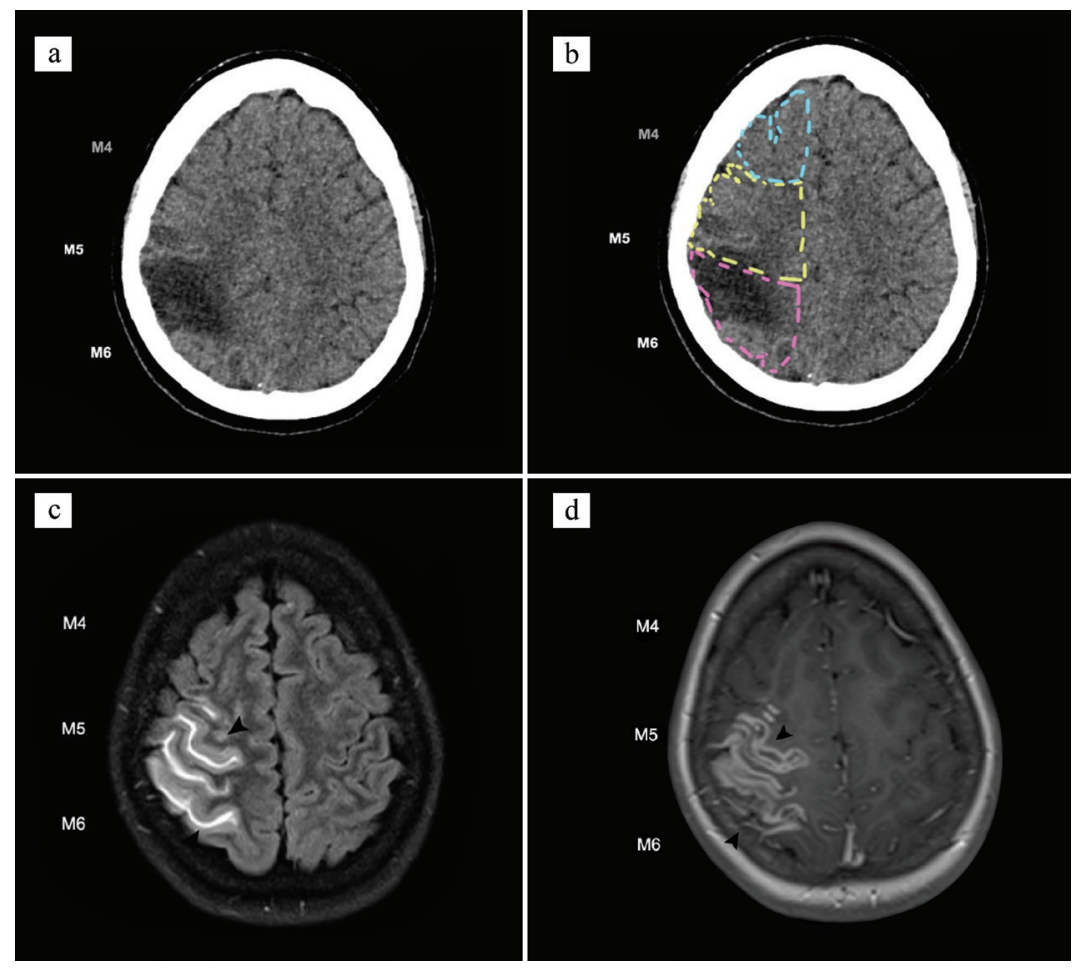

Fig. 1 a-d. Axial image of non-contrast CT Brain (FIG 1a,b) taken at the supraganglionic level showing ill-defined hypodensity involving the M5 and M6 region of the right middle cerebral artery (MCA) territory with associated sulcal effacement. MRI brain in FLAIR sequence (FIG 1c) and T1W post gadolinium administration (FIG. 1d) showed leptomeningeal enhancement and hyperintense signal of the right paracentral gyri, supramarginal and angular gyri (arrowheads) giving rise to ivy sign appearance likely due to engorged pia vessels with thickened arachnoid membrane.

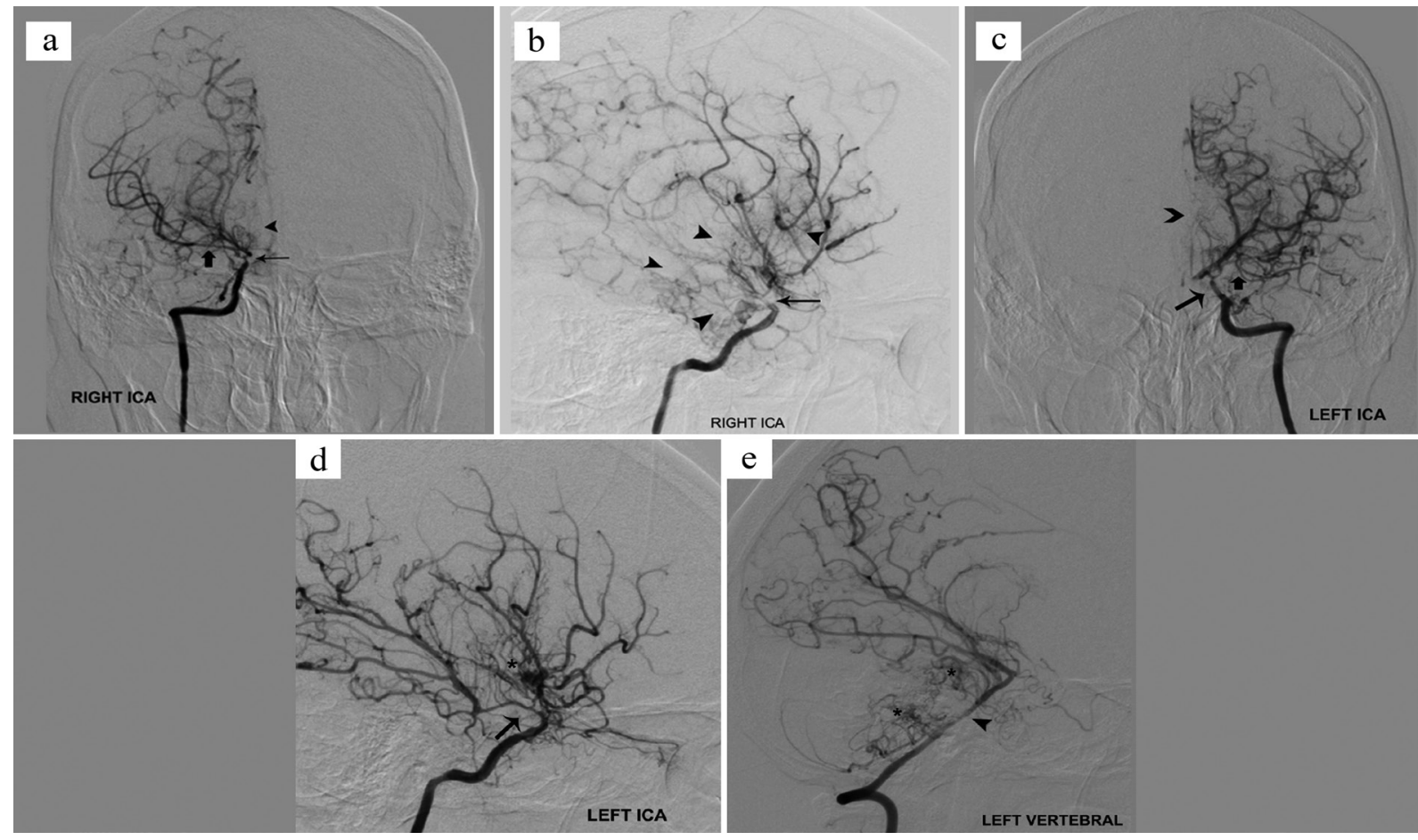

Fig. 2 a-e. Right internal carotid artery (ICA) angiography (FIG 2 a,b) showed multiple lenticulostriate collaterals giving the 'puff of smoke' appearance (arrowheads), severe stenosis of the right supraclinoid ICA and ICA bifurcation (thin arrows), and small calibre right M1 (thick arrow). Left ICA angiography (FIG 2 c,d) showed stenosis of the left supraclinoid ICA and ICA bifurcation (thin arrows), stenotic left M1 (thick arrow) and left ACA (arrowhead) with lenticulostriate collaterals giving the "puff of smoke" appearance (asterisk). Left vertebral angiogram (Figure 2e) showed stenotic V4 and basilar artery (arrowhead) with multiple collaterals of the vertebrobasilar system (asterisks). 
Patient Consent for Publication: Written informed consent was obtained from the patient.

Author Contributions: Concept - K.Y.L., C.S.K.; Supervision - H.J.T.; Materials - K.Y.L., F.S.; Data Collection and/or Processing - K.Y.L., F.S.; Analysis and/or Interpretation - K.Y.L., F.S., C.S.K.; Writing - K.Y.L.

Conflict of Interest: The authors have no conflict of interest to declare.

\section{REFERENCES}

1. Kim JS. Moyamoya disease: epidemiology, clinical features, and diagnosis. J Stroke. 2016;18:2-11. [CrossRef]

2. Lee R, Sung K, Park YM, Yu JJ, Koh YC, Chung S. A case of Moyamoya disease in a girl with thyrotoxicosis. Yonsei Med J. 2009;50:594-598. [CrossRef]

3. Im SH, Oh CW, Kwon OK, Kim JE, Han DH. Moyamoya disease associated with Graves disease: special considerations regarding clinical significance and management. J Neurosurg. 2005;102:1013-1017. [CrossRef] 